\title{
Heparin-binding epidermal growth factor-like growth factor suppresses experimental liver fibrosis in mice
}

\author{
Guangcun Huang ${ }^{1}$, Gail E Besner ${ }^{2,3,4}$ and David R Brigstock ${ }^{1,3,4,5}$
}

Heparin-binding epidermal growth factor-like growth factor (HB-EGF) is a cytoprotective agent in several organ systems but its roles in liver fibrosis are unclear. We studied the roles of HB-EGF in experimental liver fibrosis in mice and during hepatic stellate cell (HSC) activation. Thioacetamide (TAA; $100 \mathrm{mg} / \mathrm{kg}$ ) was administered by intraperitoneal injection three times a week for 4 weeks to wild-type $\mathrm{HB}-\mathrm{EGF}^{+/+}$or $\mathrm{HB}-\mathrm{EGF}-$ null $\left(\mathrm{HB}^{-\mathrm{EGF}^{-/-}}\right)$male mice. Livers were examined for histology and expression of key fibrotic markers. Primary cultured HSCs isolated from untreated HB-EGF ${ }^{+/+}$or $\mathrm{HB}_{-} \mathrm{EGF}^{-1-}$ mice were examined for fibrotic markers and/or cell migration either during culture-induced activation or after exogenous HB-EGF $(100 \mathrm{ng} / \mathrm{ml})$ treatment. TAA induced liver fibrosis in both $\mathrm{HB}^{-\mathrm{EGF}^{+/+}}$and $\mathrm{HB}^{-\mathrm{EGF}^{-/}}$mice. Hepatic HB-EGF expression was decreased in TAA-treated HB-EGF ${ }^{+/+}$mice by $37.6 \%(P<0.05)$ as compared with animals receiving saline alone. $\mathrm{HB}-\mathrm{EGF}^{-/}$mice treated with TAA showed increased hepatic $\alpha$-smooth muscle actin-positive cells and collagen deposition, and, as compared with $\mathrm{HB}^{-E_{\text {GFF }}+/+}$ mice, TAA-stimulated hepatic mRNA levels in $\mathrm{HB}_{-E^{-1-}}$ mice were, respectively, 2.1-, 1.7-, 1.8-, 2.2-, 1.2- or 3.3-fold greater for $\alpha$-smooth muscle actin, $\alpha 1$ chain of collagen I or III (COL1A1 or COL3A1), transforming growth factor- $\beta 1$, connective tissue growth factor or tissue inhibitor of metalloproteinase-1 $(P<0.05)$. HB-EGF expression was detectable in primary cultured HSCs from HB-EGF ${ }^{+/+}$mice. Both endogenous and exogenous HB-EGF inhibited HSC activation in primary culture, and HB-EGF enhanced HSC migration. These findings suggest that HB-EGF gene knockout in mice increases susceptibility to chronic TAA-induced hepatic fibrosis and that HB-EGF expression or action is associated with suppression of fibrogenic pathways in HSCs. Laboratory Investigation (2012) 92, 703-712; doi:10.1038/labinvest.2012.3; published online 13 February 2012

KEYWORDS: connective tissue growth factor; HB-EGF; heparin-binding epidermal growth factor-like growth factor; hepatic stellate cells; liver fibrosis

Heparin-binding epidermal growth factor (EGF)-like growth factor (HB-EGF), a member of the EGF superfamily, is expressed by many cell types and is produced in multiple tissues such as lung, skeletal muscle, brain and heart. ${ }^{1,2}$ HB-EGF participates in a variety of physiological and pathological processes including development, wound healing, blastocyst implantation, atherosclerosis and tumor formation. ${ }^{2}$ Two recently established strains of HB-EGF knockout $\left(\mathrm{HB}-\mathrm{EGF}^{-1-}\right)$ mice $^{3,4}$ have a relatively severe phenotype with a high incidence of neonatal death. ${ }^{5}$ Surviving HB-EGF ${ }^{-1-}$ mice, however, grow normally and are fertile, but have a significantly shorter life span and exhibit defects that include enlarged and dysfunctional hearts, heart valve malformation including enlarged semilunar and atrioventricular valves, and thickened mesenchymal tissue and alveolar immaturity in the lungs. ${ }^{6}$ Impaired remodeling of $\mathrm{HB}-\mathrm{EGF}^{-1-}$ valves was not due to decreased apoptosis but rather to excessive proliferation of mesenchymal cells. ${ }^{3}$ In another conditional HB-EGF knockout mice, skin wound healing was retarded due to impaired keratinocyte migration. ${ }^{7,8}$ On the other hand, overexpression of HB-EGF in pancreatic islets is associated with intra-islet fibrosis. ${ }^{9,10}$

Transgenic overexpression of HB-EGF in hepatocytes did not change liver/body weight ratios or the ratio of proliferating hepatocytes in adult mice but these parameters were both greater in transgenic mice after partial

\footnotetext{
${ }^{1}$ The Research Institute at Nationwide Children's Hospital, Center for Clinical and Translational Research, Columbus, OH, USA; ${ }^{2}$ Center for Perinatal Research, The Research Institute at Nationwide Children's Hospital, Columbus, OH, USA; ${ }^{3}$ Department of Pediatric Surgery, Nationwide Children's Hospital, Columbus, OH, USA;

${ }^{4}$ Department of Surgery, The Ohio State University, Columbus, OH, USA and ${ }^{5}$ Department of Molecular and Cellular Biochemistry, The Ohio State University, Columbus, $\mathrm{OH}, \mathrm{USA}$

Correspondence: Dr DR Brigstock, PhD, The Research Institute at Nationwide Children's Hospital, Center for Clinical and Translational Research, Research II, Room WA 2020, 700 Children's Dr, Columbus, OH 43205, USA.

E-mail: David.Brigstock@NationwideChildrens.org

Received 7 March 2011; revised 29 November 2011; accepted 18 December 2011
} 
hepatectomy compared with wild-type mice, supporting a role for HB-EGF in liver regeneration. ${ }^{11}$ In addition, hepatic expression of HB-EGF or transforming growth factor $\alpha$ (TGF- $\alpha$ ) were increased after acute carbon tetrachloride $\left(\mathrm{CCl}_{4}\right)$ injury in rats, ${ }^{12,13}$ with Kupffer cells and sinusoidal endothelial cells being the prominent producers of HB-EGF. Kiso et $\mathrm{al}^{14}$ demonstrated that some hepatocytes in cirrhotic rat liver were positive for HB-EGF expression leading to the suggestion that ectopic expression of HB-EGF is associated with hepatocyte transformation during hepatocarcinogenesis.

As stated above, previous studies were designed to determine the role of HB-EGF in driving hepatocyte proliferation after acute injury or during tumorigenesis rather than to elucidate its potential contribution to pathways of hepatic fibrosis, especially in non-parenchymal liver cells. For example, there have been no published studies regarding the role of HB-EGF in the liver fibrogenesis or activation of hepatic stellate cells (HSCs), the latter of which have a key role in the development of liver fibrosis through their overt deposition of extracellular matrix components in response to the combined actions of transforming growth factor $\beta 1$ (TGF- $\beta 1$ ) and its downstream mediator, connective tissue growth factor (also known as CCN2). ${ }^{15-21}$ In the present studies, we have investigated the role of HB-EGF in HSC activation and liver fibrosis, including its modulation of TGF- $\beta 1$ or CCN2 expression. We show that HB-EGF gene knockout in mice increases susceptibility to hepatic fibrosis in response to chronic liver injury induced by thioacetamide (TAA) or $\mathrm{CCl}_{4}$, and that HB-EGF expression or action is associated with suppression of fibrogenic pathways in HSCs. These findings reveal a novel role of HB-EGF in HSCs activation and liver fibrosis, and suggest that HB-EGF has potential therapeutic value for treating liver fibrosis.

\section{MATERIALS AND METHODS \\ Mice}

$\mathrm{HB}^{-\mathrm{EGF}^{-1-}}$ and HB-EGF ${ }^{+/+}$mice on a mixed C57BL/6J X 129/Sv background (B6; 129-Hbegf $\left.f^{t m 1 D c l} / \mathrm{Mmnc}\right)$ were a kind gift from Dr David Lee (Chapel Hill, NC, USA). HB-EGF ${ }^{-l-}$ mice were created by the replacement of HB-EGF exons 1 and 2 with PGK-Neo, thus deleting the signal peptide and propeptide domains. ${ }^{4}$ The desired targeting events were verified by Southern blots of genomic DNA and exon-specific PCR, with northern blots confirming absence of the respective transcripts. All animal procedures were approved by the Institutional Animal Care and Use Committee of The Research Institute at Nationwide Children's Hospital (Columbus, OH, USA).

\section{TAA Administration}

$\mathrm{HB}^{-\mathrm{EGF}^{-1-}}$ or HB-EGF${ }^{+/+}$male mice, 6-8 weeks of age, received intraperitoneal injection of either $100 \mathrm{mg} / \mathrm{kg}$ TAA (Sigma-Aldrich, St Louis, MO, USA) in saline or saline alone three times per week for 4 weeks, and were killed $72 \mathrm{~h}$ after the last injection. After sacrifice, liver tissues were harvested either immediately and snap-frozen in liquid nitrogen for subsequent hepatic RNA extraction, or fixed with $4 \%$ paraformaldehyde (Sigma-Aldrich) for histological analysis.

\section{$\mathrm{CCl}_{4}$ Administration}

$\mathrm{HB}_{\mathrm{EGF}}{ }^{-1-}$ or $\mathrm{HB}^{-\mathrm{EGF}^{+/+}}$male mice received intraperitoneal injection of $0.5 \mu \mathrm{lCCl}_{4}$ (Sigma-Aldrich) in $29.5-\mu \mathrm{l}$ vegetable oil or $30-\mu \mathrm{l}$ vegetable oil alone three times per week for 5 weeks. Seventy-two hours after the last injection, mice were killed and livers were processed for histological evaluation.

\section{Histology}

Liver tissues were fixed with $4 \%$ paraformaldehyde in phosphate-buffered saline for $18 \mathrm{~h}$ and then embedded in paraffin. Sections of $5 \mu \mathrm{m}$ thickness were cut and stained with hematoxylin-eosin (H\&E). For immunohistochemical detection of alpha-smooth muscle actin ( $\alpha$-SMA), slides were incubated with monoclonal mouse anti- $\alpha$-SMA IgG (Dako, Glostrup, Denmark) followed by development with UltraTek reagents and AEC Chromogenic Substrates (all from ScyTek Laboratories, Logan, UT, USA) and hematoxylin counterstain. Collagen was detected by staining sections with $0.1 \%$ Sirius Red (Sigma-Aldrich). Photomicrographs were recorded with a Zeiss AxioCam HR Camera attached to a Zeiss Axioskop microscope (Carl Zeiss Vision GmbH, Germany). Protein staining for $\alpha$-SMA or collagen was analyzed with NIH image software ImageJ (version 1.34, US National Institute of Health).

\section{Isolation and Culture of Mouse Primary HSCs or Hepatocytes}

HSCs were isolated from the livers of untreated HB-EGF ${ }^{-1-}$ or HB-EGF ${ }^{+1+}$ male mice, $6-8$ weeks of age, as previously described by us. ${ }^{17}$ HSCs were harvested by gradient centrifugation using OptiPrep (Invitrogen, Carlsbad, CA, USA), resuspended in Dulbecco's minimum essential medium (DMEM)/F-12 (Mediatech, Manassas, VA, USA) containing $20 \%$ fetal bovine serum, $100 \mathrm{U} / \mathrm{ml}$ penicillin and $100 \mathrm{mg} / \mathrm{ml}$ streptomycin (all from Mediatech), plated into plastic tissue culture dishes or tissue culture plates (Corning Incorporated, Corning, NY, USA), and maintained at $37^{\circ} \mathrm{C}$ in a humidified atmosphere of $5 \% \mathrm{CO}_{2} / 95 \%$ air. The next day, cells were replaced with fresh DMEM/F-12 containing 10\% fetal bovine serum, and medium replacement was then repeated every other day if applicable. Cells were analyzed by RT-PCR for expression of fibrotic markers after 24-h treatment with $0-100 \mathrm{ng} / \mathrm{ml}$ recombinant $\mathrm{HB}-\mathrm{EGF}^{22}$ in the presence or absence of $10 \mu \mathrm{g} / \mathrm{ml}$ Diphtheria toxin mutant CRM197 (BioAcademia, Ibaraki, Osaka, Japan), which blocks HB-EGF receptor binding and inhibits HB-EGF activity.

Hepatocytes were isolated from untreated HB-EGF WT male mice, $6-8$ weeks of age, by a modification of previously described methodology. ${ }^{23}$ Hepatocytes were resuspended in DMEM containing $10 \%$ fetal bovine serum, $100 \mathrm{U} / \mathrm{ml}$ penicillin and $100 \mathrm{mg} / \mathrm{ml}$ streptomycin, plated on $60-\mathrm{mm} \mathrm{BD}$ 
Biocoat Collagen I Culture Dishes (BD Biosciences, Bedford, MA, USA), and maintained at $37^{\circ} \mathrm{C}$ in a humidified atmosphere of $5 \% \mathrm{CO}_{2} / 95 \%$ air. Four hours later, the medium was replaced with serum-free DMEM supplemented with $7.5 \mu \mathrm{g} / \mathrm{ml}$ of hydrocortisone (Sigma-Aldrich), $1 \times$ insulintransferrin sodium selenite solution (Mediatech), $100 \mathrm{U} / \mathrm{ml}$ penicillin and $100 \mathrm{mg} / \mathrm{ml}$ streptomycin, and medium replacement was then repeated every other day if applicable.

\section{Immunofluorescence}

Cells grown on coverslips in 6-well tissue culture plates were fixed with ice-cold $100 \%$ methanol for $20 \mathrm{~min}$ at $4^{\circ} \mathrm{C}$. After blocking with $5 \%$ BSA in phosphate-buffered saline, cells were incubated with primary antibody against HB-EGF (Santa Cruz Biotechnology, Santa Cruz, CA, USA) in blocking solution for $1 \mathrm{~h}$ at $37^{\circ} \mathrm{C}$, followed by secondary Alex Fluor 488 goat anti-mouse IgG (Invitrogen) for $45 \mathrm{~min}$ at room temperature. The coverslips were mounted in $10 \%$ glycerol and photomicrographs were recorded with a Zeiss AxioCam Camera attached to a Zeiss Axiovert 25 microscope (Carl Zeiss MicroImaging GmbH, München, Germany).

\section{Cell Migration}

Primary cultured HSCs were plated into 6-well tissue culture plates in DMEM/F-12 containing 10\% fetal bovine serum and grown to confluence. After pre-incubation in DMEM/ F-12 containing $0.5 \%$ fetal bovine serum for $24 \mathrm{~h}$, the cultures were scratch-wounded with a $10-\mu$ l pipette tip, washed twice with phosphate-buffered saline, and then incubated for $24 \mathrm{~h}$ with $100 \mathrm{ng} / \mathrm{ml}$ recombinant HB-EGF in the presence or absence of $10 \mu \mathrm{g} / \mathrm{ml}$ CRM197. Photographs of the scraped area in each well were recorded using a Zeiss AxionCam Camera. Cell migration was quantified by the number of cells migrated to the scratched area after $24 \mathrm{~h}$.

\section{RNA Extraction and Reverse Transcription}

Total cellular RNA was extracted using Trizol Reagent (Invitrogen), and reverse transcribed to produce cDNA using Superscript II Reverse Transcriptase (Invitrogen) and Oligo(dT) ${ }_{12-16}$ primers (Applied Biosystems, Foster City, CA, USA), according to the manufactures' directions.

\section{PCR and Quantitative Real-Time PCR}

The primer sequences were as follows: $\alpha$-SMA: $5^{\prime}$-TTCG TTACTACTGCTGAGCGTGAGA- $3^{\prime}$ (sense) and $5^{\prime}$-AAAGAT GGCTGGAAGAGGGTC- $3^{\prime}$ (antisense) (200 bp); $\beta$-actin: $5^{\prime}$-TGTTACCAACTGGGACGACA- $3^{\prime}$ (sense) and $5^{\prime}$-CTTTT CACGGTTGGCCTTAG-3' (antisense) (130 bp); CCN2: $5^{\prime}-\mathrm{C}$ CAGGAAGTAAGGGACACGA- $3^{\prime}$ (sense) and $5^{\prime}$-GGTTCTC ACTTTGGTGGGAT-3' (antisense) (148 bp); $\alpha 1$ chain of collagen I (COL1A1): 5'-CCAAGGGTAACAGCGGTGAA- $3^{\prime}$ (sense) and $5^{\prime}$-CCTCGTTTTCCTTCTTCTCCG-3' (antisense) (124 bp); $\alpha 1$ chain of collagen III (COL3A1): $5^{\prime}$-AAC GGAGCTCCTGGCCCCAT- $3^{\prime}$ (sense) and $5^{\prime}$-CCATCACTGC CCCGAGCACC- $3^{\prime}$ (antisense) (113 bp); TGF- $\beta 1$ : $5^{\prime}$-GGTT
CATGTCATGGATGGTGC- $3^{\prime}$ (sense) and $5^{\prime}$-TGACGTC ACTGGAGTTGTACG- $3^{\prime}$ (antisense) (130 bp); and TIMP-1: $5^{\prime}$-GCATCTCTGGCATCTGGCATC- $3^{\prime}$ (sense) and $5^{\prime}$-GCGG TTCTGGGACTTGTGGGC- $3^{\prime}$ (antisense) (294 bp). Primers were validated by PCR and products were confirmed by $1.5 \%$ agarose gel electrophoresis using ethidium bromide staining. To measure gene expression, first strand cDNA products mixed with primers and SYBR Green PCR Master Mix reagent (Applied Biosystems) were subjected to quantitative real-time PCR on an Applied Biosystems 7500 Real-Time PCR System, and the cycling parameters were: $50^{\circ} \mathrm{C}$ for $2 \mathrm{~min}, 95^{\circ} \mathrm{C}$ for $10 \mathrm{~min}$, followed by 40 cycles of $95^{\circ} \mathrm{C}$ for $15 \mathrm{~s}$ and $60^{\circ} \mathrm{C}$ for $1 \mathrm{~min}$. Samples were run in triplicate, and expression values were normalized to housekeeping gene $\beta$-actin primers using the $\Delta \Delta C_{\mathrm{t}}$ method.

\section{Statistical Analysis}

Data are presented as mean \pm s.d. Statistical analysis was performed using ANOVA one-way test, with $P<0.05$ considered to be statistically significant.

\section{RESULTS}

\section{Enhanced Susceptibility of HB-EGF ${ }^{-1-}$ Mice to Liver Fibrosis Induced by Chronic Injury}

No differences in liver histology were evident between HB$\mathrm{EGF}^{-1-}$ and $\mathrm{HB}-\mathrm{EGF}^{+/+}$mice treated with saline alone (Figure 1a). Compared with these controls, chronic administration of TAA induced liver fibrosis in either $\mathrm{HB}_{-\mathrm{EGF}^{+/+}}$ or $\mathrm{HB}_{-\mathrm{EGF}^{-1-}}$ mice (Figure 1), as shown by a 1.7- or 3.6-fold increase, respectively, in collagen deposition $(P<0.05$; Figure $1 \mathrm{~d}$ and Supplementary Table S1) and a 3.3- or 10.6-fold increase, respectively, in TIMP-1 gene expression $(P<0.05$; Figure 2). After chronic TAA administration in $\mathrm{HB}^{-\mathrm{EGF}^{+1+}}$ mice, hepatic HB-EGF gene expression decreased by $37.6 \%$ $(P<0.05$; Figure 2$)$, showing that suppression of HB-EGF expression was associated with onset and/or progression of the liver fibrosis.

Chronic TAA administration resulted in a 1.9- or 1.7-fold higher level of, respectively, $\alpha$-SMA immunoreactivity $(P<0.05$; Figure 1d) or collagen staining $(P<0.05$; Figure 1d) in $\mathrm{HB}_{-\mathrm{EGF}^{-1-}}$ mice $v s \mathrm{HB}-\mathrm{EGF}^{+1+}$ mice. As compared with $\mathrm{HB}_{-\mathrm{EGF}^{+/+}}$mice, TAA-stimulated hepatic mRNA levels in $\mathrm{HB}^{-\mathrm{EGF}^{-1-}}$ mice were, respectively, 2.1-, 1.7-, 1.8-, 2.2-, 1.2- or 3.3-fold greater for $\alpha$-SMA, COL1A1, COL3A1, TGF- $\beta 1$, CCN2 or TIMP-1 (Figure 2). Collectively these data show that absence of HB-EGF gene expression in murine experimental fibrosis resulted in enhanced production of $\alpha$-SMA-positive cells, collagen deposition and expression of pro-fibrotic genes.

To verify that the inhibitory effect of HB-EGF was not restricted to TAA-induced liver fibrosis, HB-EGF WT and $\mathrm{KO}$ mice alternatively received $\mathrm{CCl}_{4}$ for 5 weeks and the livers were processed for histological evaluation. HB-EGF KO mice demonstrated more collagen deposition as shown by Sirius Red staining (Supplementary Figure S1), suggesting 

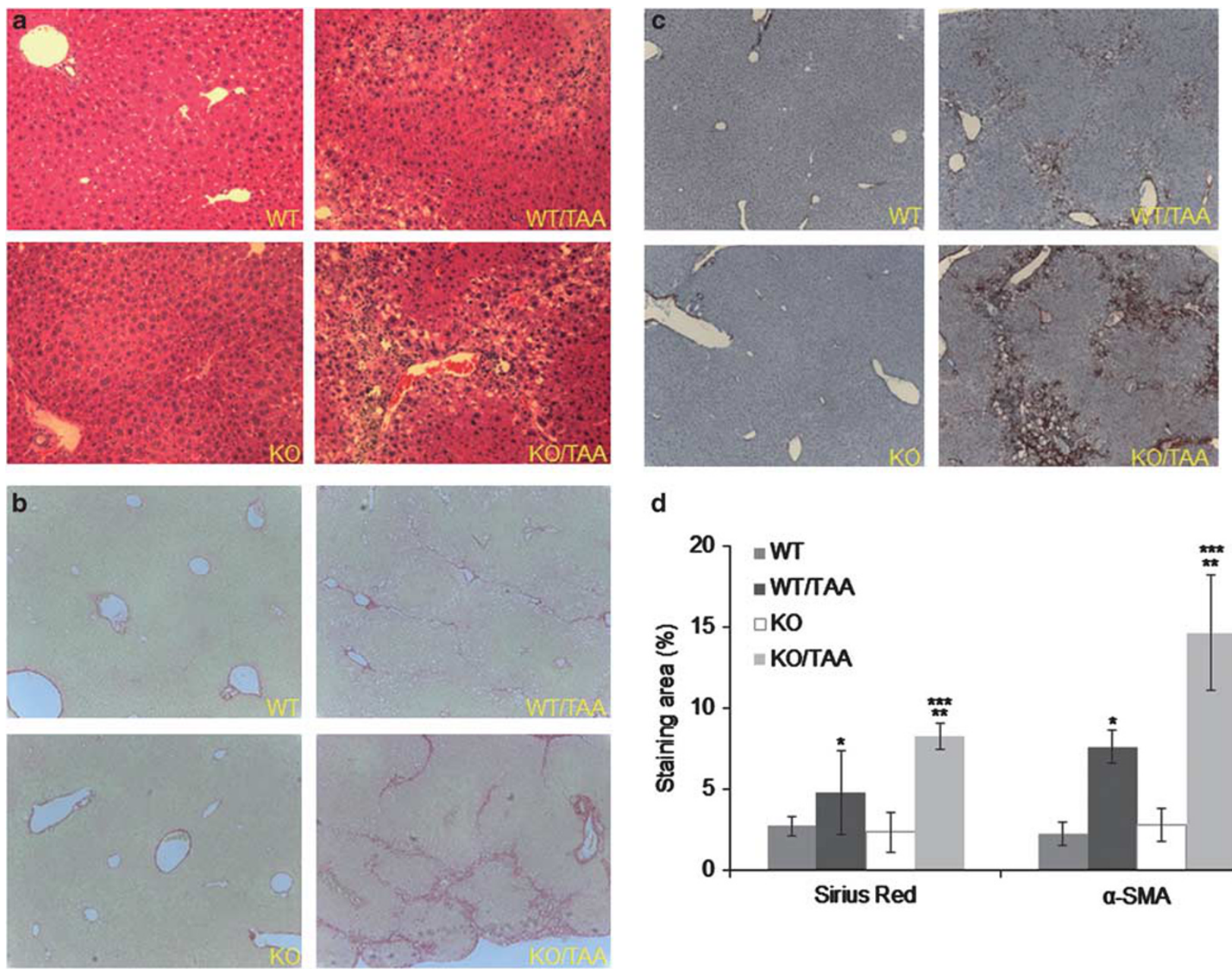

d

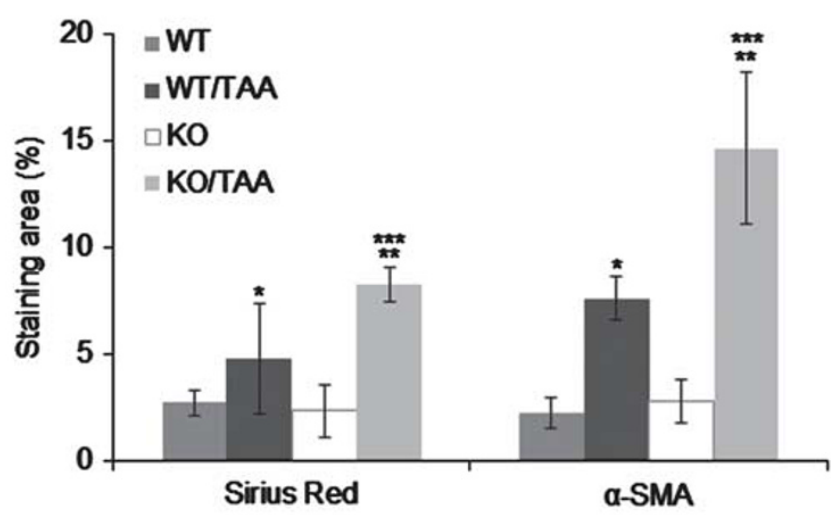

Figure 1 Histology: $\mathrm{HB}^{-\mathrm{EGF}^{+/+}}$(WT) or HB-EGF${ }^{-/-}$(KO) mice were injected with thioacetamide (TAA) three times per week for 4 weeks. Liver tissues were removed, fixed and sections of $5 \mu \mathrm{m}$ were stained with H\&E (a), Sirius Red (b), or with an $\alpha$-SMA antibody (c). Sirius Red or $\alpha$-SMA staining was analyzed with $\mathrm{NIH}$ image software ImageJ (d). Data are the mean \pm s.d. of each group $(n=3,4$ or 5$)$ with triplicate determinations. ${ }^{*} P<0.05$ vs WT, ${ }^{* * P}<0.05$ vs KO, $* * * P<0.05$ vs WT/TAA.

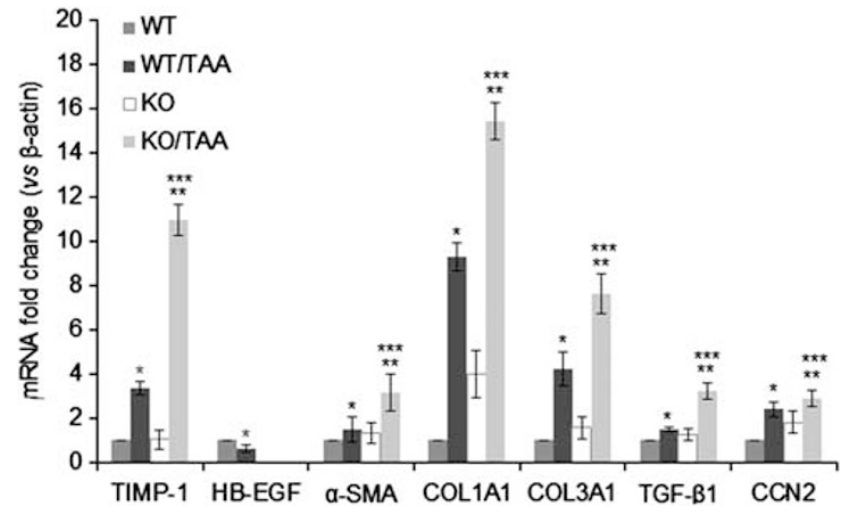

Figure 2 Fibrogenic gene expression in liver. Heparin-binding epidermal growth factor-like growth factor (HB-EGF) WT or KO mice were injected with thioacetamide (TAA) for 4 weeks, and hepatic total RNA was extracted. Samples were subjected to quantitative real-time PCR for determination of hepatic expression of TIMP-1, HB-EGF, $\alpha$-SMA, COL1A1, COL3A1, transforming growth factor- $\beta 1$ (TGF- $\beta 1$ ), or CCN2 mRNA. Data are the means \pm s.d. of each group ( $n=3,4$ or 5 ) with triplicate determinations. ${ }^{*} P<0.05$ vs $\mathrm{WT},{ }^{* *} P<0.05$ vs $\mathrm{KO},{ }^{* * *} P<0.05$ vs WT/TAA. enhanced susceptibility of $\mathrm{HB}-\mathrm{EGF} \mathrm{KO}$ mice to $\mathrm{CCl}_{4}$-induced fibrosis.

\section{Gene Expression of HB-EGF in Primary Cultures of Hepatocytes or HSCs}

Previous data have shown that HB-EGF is produced by Kupffer cells, sinusoidal endothelial cells and under-stimulated or abnormal hepatocytes. ${ }^{24,25}$ We extended these studies to show that HB-EGF was expressed by normal cultured mouse primary hepatocytes but that the level of HB-EGF mRNA was decreased by $35 \%$ after treatment of the cells with 2 ng/ml TGF- $\beta 1$ (eBioscience, San Diego, CA, USA; $P<0.05$; Figure 3). As hepatocytes are the principal hepatic cell type, this phenomenon was consistent with the overall decreased tissue expression of HB-EGF in fibrotic liver (Figure 2). On the other hand, as HSCs have a pivot role in fibrogenesis even though they are present in the liver at much lower frequency than hepatocytes, we also examined the role of HB-EGF in HSC function. To do this, HSCs were isolated from untreated 


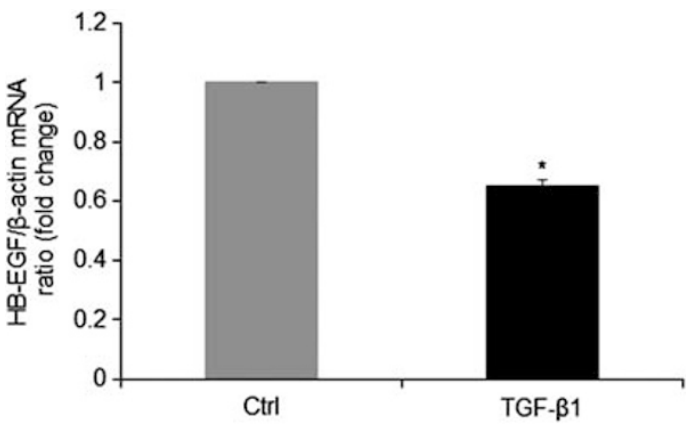

Figure 3 Effect of exogenous recombinant transforming growth factor- $\beta 1$ (TGF- $\beta 1$ ) on heparin-binding epidermal growth factor-like growth factor (HB-EGF) gene expression in primary cultured hepatocytes. Hepatocytes isolated from untreated HB-EGF WT mice were cultured for 2 days. Cells were pre-incubated in Dulbecco's minimum essential medium (DMEM) containing $0.5 \%$ fetal bovine serum for $24 \mathrm{~h}$, followed by treatment of the cells with $2 \mathrm{ng} / \mathrm{ml}$ recombinant human TGF- $\beta 1$ for an additional $24 \mathrm{~h}$. RNA was isolated and analyzed by quantitative real-time PCR to determine HBEGF expression. Data are the mean \pm s.d. of three experiments with triplicate determinations. ${ }^{*} P<0.05$ vs control.

HB-EGF ${ }^{+1+}$ male mice at 6-8 weeks of age and allowed to grow in culture for up to 5 days. HSC cultures demonstrated typical characteristics as we have previously reported ${ }^{17}$ and also stained positively for glial fibrillary acidic protein, a wellcharacterized HSC marker (Supplementary Figure S2). As shown in Figure 4a, HB-EGF mRNA expression increased significantly during the first 5 days of culture, corresponding to the period during which the cells autonomously selfactivated and acquired an $\alpha$-SMA-positive myofibroblastic phenotype (data not shown). HB-EGF mRNA expression levels were correlated with those of CCN2 mRNA, including a transient decrease in expression on day 4 (Figures $4 \mathrm{a}$ and d). By day 5 of culture, the presence of low levels of HB-EGF protein in the cells was demonstrable by immunofluorescence (Figure 4e). Expression of mRNA for $\alpha$-SMA or COL1A1 increased on successive days over the same time period (Figures 4b, c and Supplementary Table S2).

\section{HB-EGF Inhibits $\alpha$-SMA or COL1A1 Expression in Primary Cultured HSCs}

When HSCs were cultured for 5 days, the cells from untreated HB-EGF ${ }^{-1-}$ mice expressed higher mRNA levels for $\alpha$-SMA (Figure $4 \mathrm{~b}$ ), COL1A1 (Figure 4c) or CCN2 (Figure 4d) than those from untreated $\mathrm{HB}-\mathrm{EGF}^{+1+}$ mice, showing that endogenous HB-EGF expression suppresses HSC activation and fibrogenesis $(P<0.05)$. Additionally, expression of $\alpha$-SMA or COL1A1 was significantly decreased by treatment of day 3 HSCs from either untreated B6;129-Hbegf $f^{t m 1 D c l} / \mathrm{Mmnc}_{\mathrm{HB}-\mathrm{EGF}^{+/+}}$ mice (Figure $5 b$ ) or healthy male wild-type $\mathrm{Balb} / \mathrm{c}$ mice (Figure 5a) with recombinant HB-EGF $(P<0.05)$, although this treatment did not significantly alter $\mathrm{CCN} 2$ gene expression in the same cells. Importantly, the inhibitory effect of HB-EGF on $\alpha$-SMA or COL1A1 gene expression was reversed by $10 \mu \mathrm{g} / \mathrm{ml}$
CRM197, a specific HB-EGF inhibitor (Figure 5c). Incubation of $\mathrm{HB}_{-\mathrm{EGF}^{+1+}} \mathrm{HSCs}$ with exogenous recombinant HB-EGF inhibited endogenous HB-EGF gene expression by $60.4 \%(P<0.05$; Figure 6$)$.

\section{HB-EGF Enhances HSC Migration}

Scratch wounding of confluent HSCs resulted in a reproducibly exposed area into which the remaining cells could migrate. While a few cells migrated into this zone over the next $24 \mathrm{~h}$, the rate of HSC migration was significantly stimulated by the addition of $100 \mathrm{ng} / \mathrm{ml}$ recombinant HB-EGF and this effect was blocked in the presence of $10 \mu \mathrm{g} / \mathrm{ml}$ CRM197 (Figure 7).

\section{DISCUSSION}

The EGF family comprises EGF, TGF- $\alpha$, HB-EGF, amphiregulin (AR) and betacellulin, the latter three of which contain unique structural components that allows the proteins to bind to heparin-like molecules on cell surfaces in addition to the classic avian erythroblastosis oncogene B-type EGF receptors. Collectively, these proteins have fundamental roles in development, cell proliferation, differentiation and tumor formation. HB-EGF was first identified in the conditioned medium of cultured human macrophages and is synthesized as a 208-residue bioactive transmembrane precursor protein (proHB-EGF), which undergoes extracellular proteolytic cleavage to yield a $14-20-\mathrm{kDa}$ soluble growth factor (sHBEGF). ${ }^{26-28}$ HB-EGF is produced in multiple cell types in response to hypoxia or tissue damage and it acts as a potent mitogen and chemoattractant. ${ }^{29}$

HB-EGF expression is increased in hepatic endothelial cells or Kupffer cells following acute exposure to hepatotoxins, ${ }^{11-13}$ while its production by hepatocytes is increased after partial hepatectomy or in biopsies of human hepatocellular carcinoma, ${ }^{2,30}$ and its enhanced expression has been associated with the development or progression of human and rat hepatocarcinoma. ${ }^{14}$ Although these previous reports linked increased hepatic HB-EGF expression to either wound healing or tumorigenesis, studies of the role of HB-EGF in hepatic fibrosis have not previously been reported. In addressing this question in these studies, we found that the well-documented model of TAA-induced liver fibrosis was actually associated with a $35 \%$ decrease in hepatic HB-EGF gene expression in wild-type mice and that, further, expression of HB-EGF by primary cultured hepatocytes was inhibited by TGF- $\beta 1$, the latter of which is a well-characterized inhibitor of epithelial function in addition to acting as a stimulator of fibrogenic pathways in mesenchymal cells. Although hepatic HB-EGF expression was readily detected by RT-PCR of isolated RNA, both HB-EGF mRNA and protein levels were present at low levels in wild-type mice (and even less so in fibrotic animals) and were beyond our technical abilities to detect by, respectively, in situ hybridization or immunohistochemistry (data not shown). Nonetheless, the knockout model provided important confirmatory data in as much that, as assessed by 

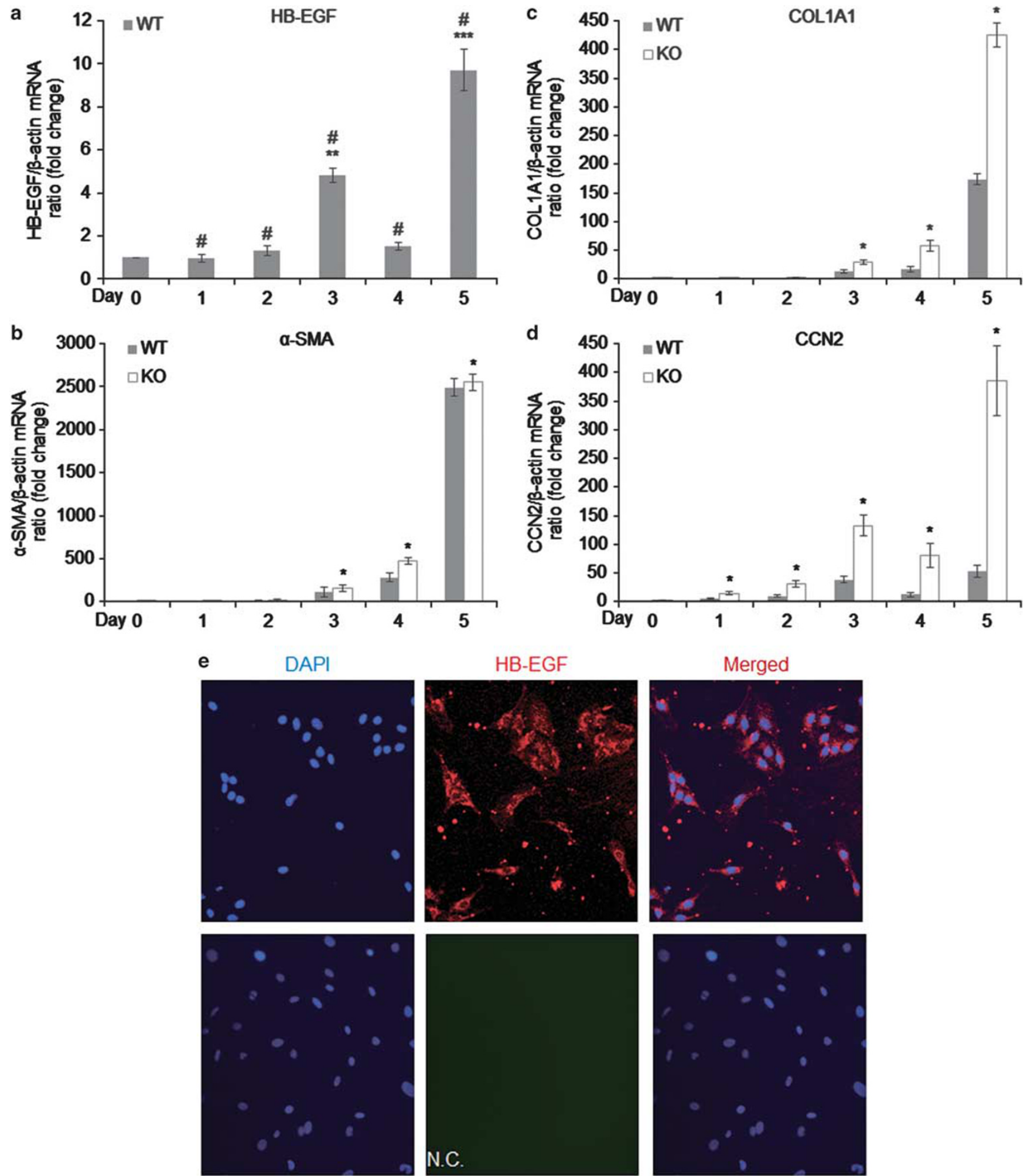

Figure 4 Fibrogenic gene expression in primary cultured hepatic stellate cells (HSCs). Stellate cells isolated from untreated heparin-binding epidermal growth factor-like growth factor (HB-EGF) WT or KO mice were cultured for 5 days. Total cellular RNA extracted from each day of culture was subjected to quantitative real-time PCR to determine expression of HB-EGF (a), $\alpha$-SMA (b), COL1A1 (c) or CCN2 (d). Wild-type cells grown on coverslips for 5 days were fixed and processed to detect HB-EGF protein by immunofluorescence (e). Data are the mean \pm s.d. of three experiments with triplicate determinations. ${ }^{\#} P<0.05$ vs day $0 ;{ }^{* * P}<0.05$ vs day 1 or day $2 ;{ }^{* *} P<0.05$ vs day $1,2,3$ or Day $4 ;{ }^{*} P<0.05$ vs WT. N.C., negative control or without anti-HB-EGF antibody. 

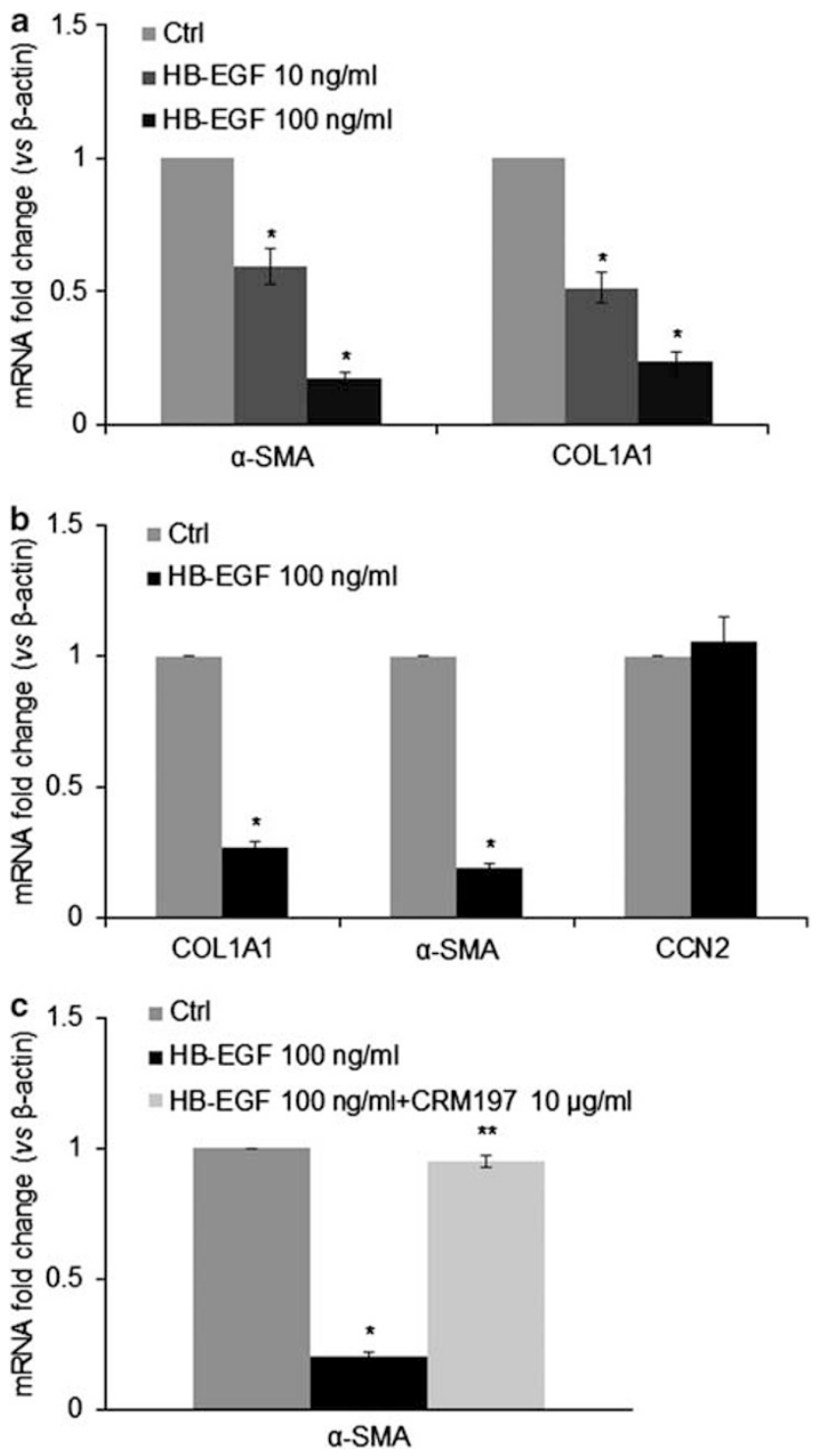

Figure 5 Effect of heparin-binding epidermal growth factor-like growth factor (HB-EGF) on fibrogenic gene expression in hepatic stellate cell (HSC) primary culture. Stellate cells isolated from untreated healthy Balb/c wildtype mice (a) or B6;129-Hbegf ${ }^{t m 1 D c l} / \mathrm{Mmnc}$ HB-EGF WT mice $(\mathbf{b}, \mathbf{c})$ were cultured for 3 days. Cells were pre-incubated in Dulbecco's minimum essential medium (DMEM)/F-12 containing $0.5 \%$ fetal bovine serum for $24 \mathrm{~h}$, followed by treatment with recombinant human HB-EGF for an additional $24 \mathrm{~h}$ with (c) or without (a, b) $10 \mu \mathrm{g} / \mathrm{ml} \mathrm{CRM197}$ before the analysis of mRNA expression of $\alpha$-SMA, COL1A1 or CCN2 by quantitative real-time PCR. Data are the mean \pm s.d. of three experiments with triplicate determinations. ${ }^{\star} P<0.05$ vs control $(\mathrm{Ctrl}) ;{ }^{*} P<0.05$ vs HB-EGF.

collagen deposition, the presence of $\alpha$-SMA-positive cells, and mRNA expression of fibrogenic markers (TGF- $\beta 1$, CCN2, $\alpha$-SMA, COL1A1, COL3A1, TIMP-1), chronic TAA administration caused more severe liver fibrosis in HB-EGF knockout mice than their wild-type counterparts. Taken together, these data showed that total hepatic HB-EGF mRNA expression was reduced during experimental fibrosis and that the fibrotic response was exacerbated in the absence

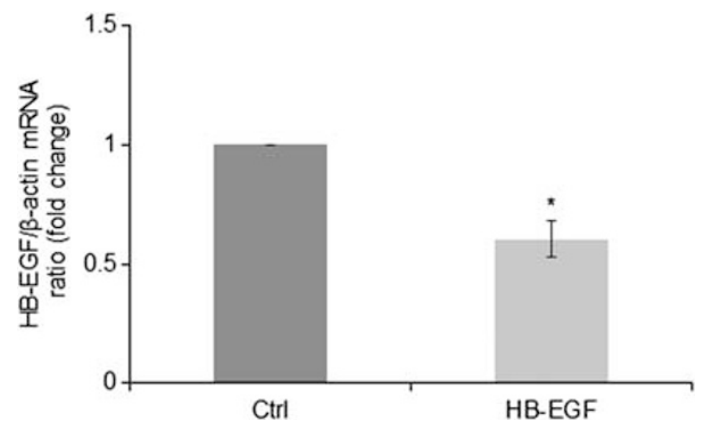

Figure 6 Effect of exogenous recombinant heparin-binding epidermal growth factor-like growth factor (HB-EGF) on endogenous HB-EGF gene expression in primary cultured hepatic stellate cells (HSCs). Stellate cells isolated from untreated HB-EGF WT mice were cultured for 3 days. Cells were pre-incubated in Dulbecco's minimum essential medium (DMEM)/F-12 containing $0.5 \%$ fetal bovine serum for $24 \mathrm{~h}$, followed by treatment with $100 \mathrm{ng} / \mathrm{ml}$ recombinant human HB-EGF for an additional $24 \mathrm{~h}$. HB-EGF mRNA expression was analyzed by quantitative real-time PCR. Data are the mean \pm s.d. of three experiments with triplicate determinations. ${ }^{*} P<0.05$ vs control (Ctrl).

of HB-EGF. These findings are consistent with one another and indicate that hepatic HB-EGF expression is associated with a suppression of liver fibrosis.

The association between HB-EGF and fibrosis is novel and was thus investigated further in HSCs, as this cell type has a key role in fibrogenic pathways. During chronic injury, HSCs differentiate into $\alpha$-SMA-positive cells that are perpetually activated and unrelentingly deposit fibrillar collagens and other extracellular matrix components in the interstitial spaces, resulting in accumulation of scar tissue and loss of hepatic function. ${ }^{15,20}$ Our studies showed that HSCs are a bone fide source of HB-EGF and that during culture-induced activation, HSC expression of HB-EGF mRNA increases as does that of fibrosis-related markers such as $\alpha$-SMA, COL1A1 and CCN2. ${ }^{17}$ Although this pattern of increased HB-EGF expression during HSC activation might appear to contradict the finding of decreased HB-EGF expression in fibrotic liver in vivo, we further showed that culture-induced mRNA expression of $\alpha$-SMA, COL1A1 or CCN2 was higher in HB$\mathrm{EGF}^{-1-}$ HSCs as compared with HB-EGF ${ }^{+1+}$ HSCs. These results suggest that under normal circumstances, HB-EGF acts to dampen activation of fibrogenic pathways in HSCs. Indeed, this possibility was supported by the finding that addition of recombinant HB-EGF to day 3 HSCs from wildtype mice resulted in an inhibition of $\alpha$-SMA or COL1A1 mRNA production. Thus, even though total hepatic HB-EGF expression decreases after fibrosing injury in vivo and activation of HSCs in vitro is associated with increased HB-EGF expression, the results of gene knockout unequivocally establish hepatic HB-EGF as a natural suppressor of the fibrotic response in as much as (i) the severity of fibrosis was greater in $\mathrm{HB}_{-} \mathrm{EGF}^{-1-}$ mice; (ii) activated HSCs from HB-EGF ${ }^{-1-}$ mice demonstrated enhanced $\alpha$-SMA or collagen mRNA production; and (iii) HSC activation and collagen production 

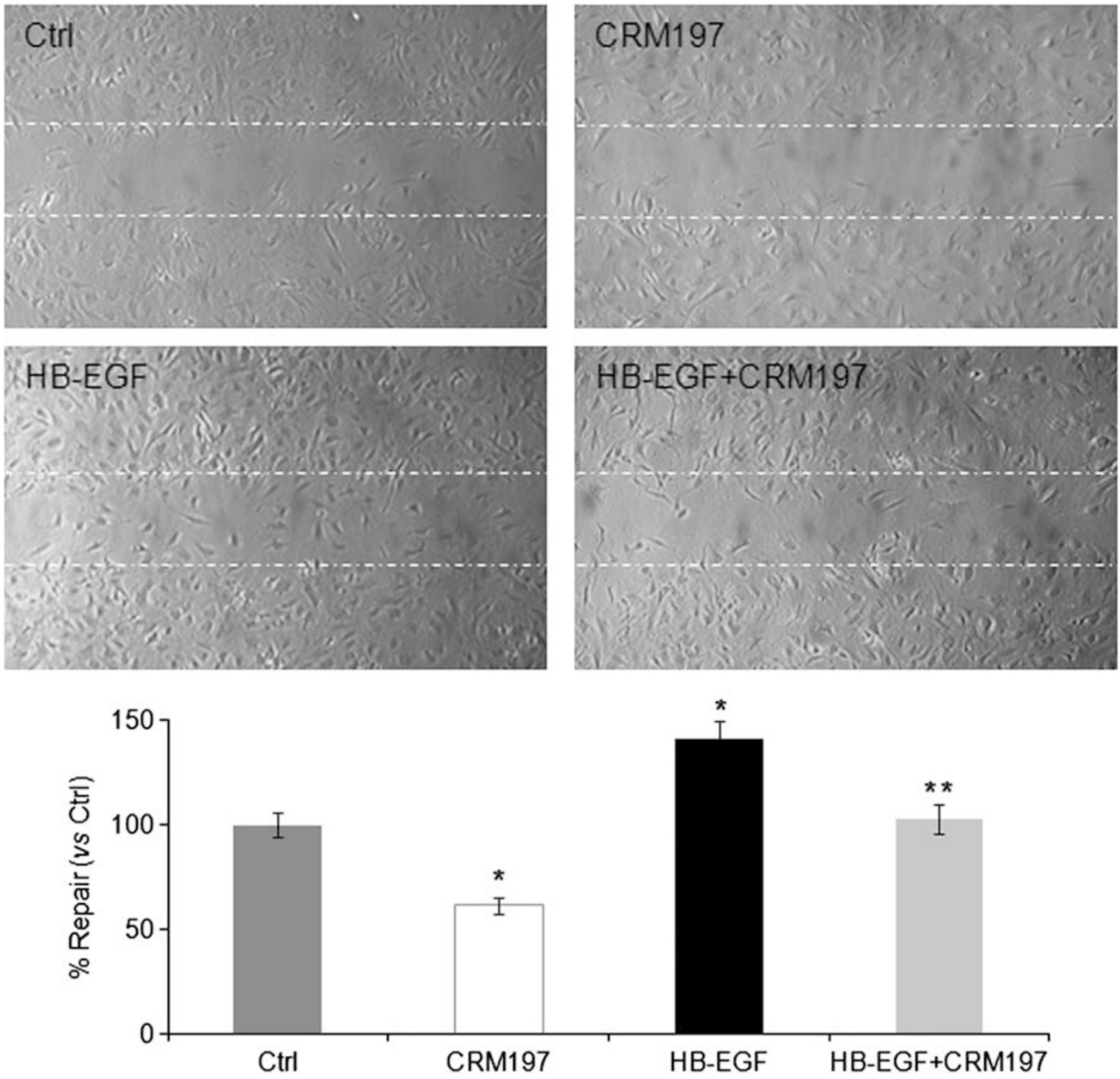

Figure 7 Effect of exogenous recombinant heparin-binding epidermal growth factor-like growth factor (HB-EGF) on hepatic stellate cell (HSC) migration. Primary cultured HSCs isolated from HB-EGF WT mice were cultured for 5 days, detached by digestion with trypsin, and plated into 6-well tissue culture plates. After pre-incubation in Dulbecco's minimum essential medium (DMEM)/F-12 containing $0.5 \%$ fetal bovine serum for $24 \mathrm{~h}$, the cultures were scratch-wounded with a $10-\mu \mathrm{l}$ pipette tip, and incubated with $100 \mathrm{ng} / \mathrm{ml}$ recombinant HB-EGF with or without $10 \mu \mathrm{g} / \mathrm{ml} \mathrm{CRM197}$ for an additional $24 \mathrm{~h}$. The bar chart shows the number of cells in the scratched area (\% vs control). Each bar represents mean \pm s.d. from three experiments.

Photomicrographs are representative of three experiments. Dotted line indicates wound margin. ${ }^{\star} P<0.05$ vs Ctrl; ${ }^{* *} P<0.05$ vs HB-EGF. Ctrl, control.

were inhibited by exposure of the cells to exogenous HB-EGF. Finally, CRM197, a specific inhibitor of HB-EGF, can reverse the effect of HB-EGF on HSC activation.

Although HB-EGF was inhibitory for HSC $\alpha$-SMA or collagen mRNA production, we demonstrated that exogenous HB-EGF stimulated HSC migration. Thus, it is plausible that the increased hepatic HB-EGF production and release by hepatocytes or Kupffer cells reported in previous tissue injury studies $^{11-13,25,26}$ serves not only as a direct stimulus for parenchymal cell proliferation but also as chemotactic stimulus for HSCs, which allows them to migrate to sites of injury and participate in the wound healing process.
We propose that as a fibrotic environment subsequently develops, HB-EGF may then serve to limit the extent or degree of fibrosis through its ability to attenuate activation and fibrogenesis in HSCs, a possibility that is supported by the finding that HB-EGF reduced basal or TGF- $\beta 1$ stimulated $\alpha$-SMA expression in the human MRC-5 fibroblast cell line. ${ }^{31}$ Thus, spatiotemporal HB-EGF gene regulation and protein production may be very critical to restoration of normal hepatic structure and function after liver injury, as shown in other organ systems. ${ }^{31,32}$

Of interest were the findings that basal or TAA-induced $\mathrm{CCN} 2$ expression was higher in livers from $\mathrm{HB}_{-} \mathrm{EGF}^{-1-}$ mice as 
compared with those from $\mathrm{HB}-\mathrm{EGF}^{+/+}$mice, and that CCN2 expression was significantly greater in $\mathrm{HB}_{-} \mathrm{EGF}^{-/-} \mathrm{HSCs}$ as compared with $\mathrm{HB}^{-\mathrm{EGF}^{+1+}}$ HSCs during culture-induced activation of the cells. These data show that endogenous HBEGF production is coupled to CCN2 production in HSCs. This is further supported by the correlation in expression between HB-EGF and CCN2 during culture-induced activation including a reproducible but as yet unexplained decrease in expression for both molecules on day 4 (see Figures $4 \mathrm{a}$ and $\mathrm{b}$ ). Over this same time-frame, $\alpha$-SMA or collagen expression continued to rise (Figures $4 \mathrm{c}$ and d) suggesting that certain time-points during HSC activation or fibrogenesis may be less dependent on CCN2 than others. This notion is consistent with previous studies in human fibroblasts that documented both CCN2-dependent and -independent mechanisms of collagen expression $^{33,34}$ as well as the recognition that CCN2 functions in a highly complex and contextual manner to drive fibrogenic pathways. ${ }^{35,36}$ Whereas CCN2 was dependent on endogenous HB-EGF, exogenously added HB-EGF on the other hand did not regulate $\mathrm{CCN} 2$ production even though it was effective in inhibiting $\alpha$-SMA or collagen expression. These results suggest either that HB-EGF-mediated CCN2 suppression in HSC changes in accordance with, but does not cause, concomitant suppression in $\alpha$-SMA or collagen expression, or that the distinct actions of exogenous $v s$ endogenous HB-EGF in suppression of $\mathrm{CCN} 2$ expression point to fundamentally discrete signaling pathways for each form of HB-EGF. Indeed the ability of endogenous membrane-bound proHB-EGF to bind and activate signal-transducing receptors on adjacent cells in a juxtracrine fashion has been well documented and is distinct from the actions of the soluble form of HB-EGF. Since, in our studies, exogenous sHB-EGF inhibited endogenous HB-EGF gene expression in $\mathrm{HB}^{-\mathrm{EGF}^{+/+}} \mathrm{HSCs}, \mathrm{CCN} 2$ production may be more directly regulated by endogenous HB-EGF than exogenous HB-EGF. Hence, although the precise mechanisms underlying HB-EGF action and $\mathrm{CCN} 2$ production have yet to be elucidated, our data are supportive of differential signaling and functions of sHB-EGF and proHB-EGF in HSCs, as is apparent in other cell types. ${ }^{7,37-40}$ A similar explanation may underlie the relatively small (but significant) difference in $\alpha$-SMA expression between HB-EGF ${ }^{+1+}$ HSCs $v s$ HB-EGF ${ }^{-1-}$ HSCs over the first 5 days of culture as compared with the pronounced effects of exogenous HB-EGF on $\alpha$-SMA expression in $\mathrm{HB}-\mathrm{EGF}^{+1+}$ HSCs. Thus, the role of HB-EGF in fibrosis is multifaceted and involves complex mechanisms that require further investigation.

We have previously shown that CCN2 production in HSCs is stimulated by endogenous TGF- $\beta{ }^{41,42}$ Although this stimulatory role has largely been attributed to direct effects of TGF- $\beta$ on the CCN2 promoter, ${ }^{42}$ it is also possible that TGF- $\beta$ in part exerts a suppressive effect on HB-EGF expression causing a similar enhancement in CCN2 levels as seen in $\mathrm{HB}-\mathrm{EGF}^{-1-}$ mice. Indeed, evidence for TGF- $\beta$-mediated suppression of HB-EGF expression was obtained in this study (albeit in hepatocytes) and future studies will need to address the HB-EGF-TGF- $\beta$ axis in HSCs in detail. Also, in HB$\mathrm{EGF}^{-1-}$ mice or HSCs, the lack of activation or stimulation of downstream targets of HB-EGF rather than HB-EGF itself may account for all or some of the differential responses as compared with $\mathrm{HB}_{-\mathrm{EGF}^{+1+}}$ mice or HSCs, a possibility to be addressed in follow-up studies.

Recent reports showed that overexpression of HB-EGF in pancreatic islets led to intra-islet fibrosis. ${ }^{9,10}$ Further studies are needed to explain the pro-fibrotic role of HB-EGF in the pancreas $v s$ its anti-fibrotic action in the liver but this difference may reflect the use of distinct genetic models (transgenic $v s$ knockout), the presence (liver) or absence (pancreas) of a noxious insult (eg, $\mathrm{CCl}_{4}$ ), and/or distinct modes of injury or repair in the liver $v s$ the pancreas, including functional differences between the stellate cells in each organ systems. ${ }^{43-45}$

Like HB-EGF, AR contains an N-terminal heparin-binding domain, which functionally engages heparan sulfate proteoglycans on the cell surface or in extracellular matrix. Chronic administration of $\mathrm{CCl}_{4}$ induced less liver fibrosis in AR-knockout mice than in wild-type mice. ${ }^{46}$ Additionally, AR-knockout mice showed impaired proliferative responses after partial liver hepatectomy, ${ }^{5}$ exhibited signs of chronic liver damage in the absence of any noxious treatment and died faster than wild-type mice in response to lethal doses of Fas-agonist antibody ${ }^{47}$ Collectively, these data show that HBEGF and AR are functionally distinct despite their structural similarity and that the actions of these EGF family members after liver injury are complex, multifaceted and nonredundant.

In summary, the present studies indicate that HB-EGF gene knockout in mice increases susceptibility to hepatic fibrosis in response to chronic TAA-induced liver injury and that HB-EGF expression or action is associated with suppression of fibrogenic pathways in HSCs. The present studies reveal a novel role of HB-EGF in HSC function and liver fibrosis, and suggest that manipulation of HB-EGF production or action may have therapeutic value for limiting hepatic fibrosis.

Supplementary Information accompanies the paper on the Laboratory Investigation website (http://www.laboratoryinvestigation.org)

\section{ACKNOWLEDGEMENTS}

We thank Dr David Lee (Chapel Hill, NC, USA) for supplying HB-EGF KO and WT mice, and Yan Chen and Amanda Darbyshire for mouse breeding and genotyping. This work was supported by the NIH Grants R01 AA016003 (to DRB), and R01 GM61193 and R01 DK074611 (to GEB).

\section{DISCLOSURE/CONFLICT OF INTEREST}

The authors declare no conflict of interest.

1. Abraham JA, Damm D, Bajardi A, et al. Heparin-binding EGF-like growth factor: characterization of rat and mouse cDNA clones, protein domain conservation across species, and transcript expression in tissues. Biochem Biophys Res Commun 1993;190:125-133. 
2. Miyamoto S, Yagi H, Yotsumoto $F$, et al. Heparin-binding epidermal growth factor-like growth factor as a new target molecule for cancer therapy. Adv Exp Med Biol 2008;622:281-295.

3. Iwamoto R, Yamazaki S, Asakura $M$, et al. Heparin-binding EGF-like growth factor and ErbB signaling is essential for heart function. Proc Natl Acad Sci USA 2003;100:3221-3226.

4. Jackson LF, Qiu TH, Sunnarborg SW, et al. Defective valvulogenesis in HB-EGF and TACE-null mice is associated with aberrant BMP signaling. EMBO J 2003;22:2704-2716.

5. Miyamoto S, Fukami T, Yagi H, et al. Potential for molecularly targeted therapy against epidermal growth factor receptor ligands. Anticancer Res 2009;29:823-830.

6. Nishi E, Klagsbrun M. Heparin-binding epidermal growth factor-like growth factor (HB-EGF) is a mediator of multiple physiological and pathological pathways. Growth Factors 2004;22:253-260.

7. Higashiyama S, Iwabuki $\mathrm{H}$, Morimoto $\mathrm{C}$, et al. Membrane-anchored growth factors, the epidermal growth factor family: beyond receptor ligands. Cancer Sci 2008;99:214-220.

8. Shirakata Y, Kimura R, Nanba D, et al. Heparin-binding EGF-like growth factor accelerates keratinocyte migration and skin wound healing. J Cell Sci 2005;118(Part 11):2363-2370.

9. Blaine SA, Ray KC, Branch KM, et al. Epidermal growth factor receptor regulates pancreatic fibrosis. Am J Physiol Gastrointest Liver Physiol 2009;297:G434-G441.

10. Means $A L$, Ray $K C$, Singh $A B$, et al. Overexpression of heparin-binding EGF-like growth factor in mouse pancreas results in fibrosis and epithelial metaplasia. Gastroenterology 2003;124:1020-1036.

11. Kiso S, Kawata S, Tamura S, et al. Liver regeneration in heparin-binding EGF-like growth factor transgenic mice after partial hepatectomy. Gastroenterology 2003;124:701-707.

12. Kiso S, Kawata S, Tamura $S$, et al. Expression of heparin-binding EGF-like growth factor in rat liver injured by carbon tetrachloride or D-galactosamine. Biochem Biophys Res Commun 1996;220:285-288.

13. Webber EM, FitzGerald MJ, Brown Pl, et al. Transforming growth factor$\alpha$ expression during liver regeneration after partial hepatectomy and toxic injury, and potential interactions between transforming growth factor- $\alpha$ and hepatocyte growth factor. Hepatology 1993;18: 1422-1431.

14. Kiso S, Kawata S, Tamura $S$, et al. Expression of heparin-binding epidermal growth factor-like growth factor in the hepatocytes of fibrotic rat liver during hepatocarcinogenesis. J Gastroenterol Hepatol 1999;14:1203-1209.

15. Friedman SL. Stellate cells: a moving target in hepatic fibrogenesis. Hepatology 2004;40:1041-1043.

16. Friedman SL. Mechanisms of hepatic fibrogenesis. Gastroenterology 2008;134:1655-1669.

17. Huang G, Brigstock DR. Integrin expression and function in the response of primary culture hepatic stellate cells to connective tissue growth factor (CCN2). J Cell Mol Med 2011;15:1087-1095.

18. Iredale J. Defining therapeutic targets for liver fibrosis: exploiting the biology of inflammation and repair. Pharmacol Res 2008;58:129-136.

19. Rachfal AW, Brigstock DR. Connective tissue growth factor (CTGF/ CCN2) in hepatic fibrosis. Hepatol Res 2003;26:1-9.

20. Reeves HL, Friedman SL. Activation of hepatic stellate cells-a key issue in liver fibrosis. Front Biosci 2002;7:d808-d826.

21. Shi-Wen X, Leask A, Abraham D. Regulation and function of connective tissue growth factor/CCN2 in tissue repair, scarring and fibrosis. Cytokine Growth Factor Rev 2008;19:133-144.

22. Davis KM, Brigstock DR, Johnson PR, et al. Production of glycosylated heparin-binding EGF-like growth factor in HeLa cells using vaccinia virus. Protein Expr Purif 1996;8:57-67.

23. Stoehr SA, Isom HC. Gap junction-mediated intercellular communication in a long-term primary mouse hepatocyte culture system. Hepatology 2003;38:1125-1135.

24. Kiso S, Kawata S, Tamura S, et al. Role of heparin-binding epidermal growth factor-like growth factor as a hepatotrophic factor in rat liver regeneration after partial hepatectomy. Hepatology 1995;22: 1584-1590.

25. Sakuda S, Tamura S, Yamada A, et al. NF- $\kappa$ B activation in nonparenchymal liver cells after partial hepatectomy in rats: possible involvement in expression of heparin-binding epidermal growth factor-like growth factor. J Hepatol 2002;36:527-533.

26. Besner G, Higashiyama S, Klagsbrun M. Isolation and characterization of a macrophage-derived heparin-binding growth factor. Cell Regul 1990;1:811-819.

27. Davis-Fleischer KM, Besner GE. Structure and function of heparinbinding EGF-like growth factor (HB-EGF). Front Biosci 1998;3: d288-d299.

28. Higashiyama S, Abraham JA, Miller J, et al. A heparin-binding growth factor secreted by macrophage-like cells that is related to EGF. Science 1991;251:936-939.

29. El-Assal ON, Besner GE. Heparin-binding epidermal growth factor-like growth factor and intestinal ischemia-reperfusion injury. Semin Pediatr Surg 2004;13:2-10.

30. Ito $Y$, Takeda T, Higashiyama $S$, et al. Expression of heparin binding epidermal growth factor-like growth factor in hepatocellular carcinoma: an immunohistochemical study. Oncol Rep 2001;8: 903-907.

31. Kirkland G, Paizis K, Wu LL, et al. Heparin-binding EGF-like growth factor mRNA is upregulated in the peri-infarct region of the remnant kidney model: in vitro evidence suggests a regulatory role in myofibroblast transformation. J Am Soc Nephrol 1998;9:1464-1473.

32. Marikovsky M, Breuing K, Liu PY, et al. Appearance of heparin-binding EGF-like growth factor in wound fluid as a response to injury. Proc Natl Acad Sci USA 1993;90:3889-3893.

33. Ricupero DA, Rishikof DC, Kuang PP, et al. Regulation of connective tissue growth factor expression by prostaglandin $E_{2}$. Am J Physiol 1999;277(Part 1):L1165-L1171.

34. Ricupero DA, Romero JR, Rishikof DC, et al. Des-Arg ${ }^{10}$-kallidin engagement of the $B 1$ receptor stimulates type I collagen synthesis via stabilization of connective tissue growth factor mRNA. J Biol Chem 2000;275:12475-12480.

35. Brigstock DR. Connective tissue growth factor (CCN2, CTGF) and organ fibrosis: lessons from transgenic animals. J Cell Commun Signal 2010;4:1-4.

36. Cicha I, Goppelt-Struebe M. Connective tissue growth factor: contextdependent functions and mechanisms of regulation. Biofactors 2009;35:200-208.

37. Higashiyama S. Metalloproteinase-mediated shedding of heparinbinding EGF-like growth factor and its pathophysiological roles. Protein Pept Lett 2004;11:443-450.

38. Higashiyama S, Nanba D. ADAM-mediated ectodomain shedding of HB-EGF in receptor cross-talk. Biochim Biophys Acta 2005;1751: 110-117.

39. Iwamoto R, Mekada E. Heparin-binding EGF-like growth factor: a juxtacrine growth factor. Cytokine Growth Factor Rev 2000;11: 335-344.

40. Miyamoto S, Hirata M, Yamazaki A, et al. Heparin-binding EGF-like growth factor is a promising target for ovarian cancer therapy. Cancer Res 2004;64:5720-5727.

41. Chen L, Charrier AL, Leask $A$, et al. Ethanol-stimulated differentiated functions of human or mouse hepatic stellate cells are mediated by connective tissue growth factor. J Hepatol 2011;55:399-406.

42. Leask A, Chen S, Pala D, et al. Regulation of CCN2 mRNA expression and promoter activity in activated hepatic stellate cells. J Cell Commun Signal 2008;2:49-56

43. Buchholz $M$, Kestler HA, Holzmann $\mathrm{K}$, et al. Transcriptome analysis of human hepatic and pancreatic stellate cells: organ-specific variations of a common transcriptional phenotype. J Mol Med 2005;83:795-805.

44. Erkan M, Weis N, Pan Z, et al. Organ-, inflammation- and cancer specific transcriptional fingerprints of pancreatic and hepatic stellate cells. Mol Cancer 2010;9:88.

45. Zhao L, Burt AD. The diffuse stellate cell system. J Mol Histol 2007;38:53-64.

46. Perugorria MJ, Latasa MU, Nicou A, et al. The epidermal growth factor receptor ligand amphiregulin participates in the development of mouse liver fibrosis. Hepatology 2008;48:1251-1261.

47. Berasain C, Garcia-Trevijano ER, Castillo J, et al. Novel role for amphiregulin in protection from liver injury. J Biol Chem 2005; 280:19012-19020. 